\title{
Computing Defeasible Meta-Logic
}

\author{
Francesco Olivieri ${ }^{1}$, Guido Governatori ${ }^{2}$, Matteo Cristani $^{3}$, and Abdul Sattar ${ }^{1}$ \\ 1 Institute for Integrated and Intelligent Systems, Griffith University, Nathan, QLD \\ 4111, Australia \{f.oliveri,a.sattar\}@griffith.edu.au \\ 2 Data61, CSIRO, Dutton Park, QLD 4102, Australia \\ guido.governatori@data61.csiro.au \\ 3 University of Verona, Verona, 37136, Italy matteo.cristani@univr.it
}

\begin{abstract}
The use of meta-rules, i.e., rules whose content includes other rules, has been advocated to model policies and the notion of power in legal reasoning, where an agent has the power to create new norms affecting other agents. The use of Defeasible Logic (DL) to model meta-rules in the application area we just alluded to has been investigated, but not from a computational viewpoint. Our aim is to fill this gap by introducing a variant of DL, Defeasible Meta-Logic, to represent defeasible meta-theories, by proposing efficient algorithms to compute the (meta-)extensions of such theories, and by proving their computational complexity.
\end{abstract}

\section{Introduction}

We investigate the issue of efficient computation of meta-rules: rules having rules as their elements. The key idea is that a rule is a (binary) relationship between a set of conditions, and a conclusion. The meaning of such a relationship is to determine under which conditions a conclusion can be generated. Meta-rules generalise such an idea by establishing that, in addition to standard conclusions, rules themselves can be the "conclusion" (and part of the set of conditions), and new rules can hence be generated from other rules. Meta-rules (or rules with nested rules) occur frequently in real life scenarios, such as normative reasoning, policies for security systems. Very often when a set of policies is represented by a set of rules, we have to consider the policy that contains conditions (rules) about itself (or about another set of rules/policies). Consider the example in [27], where a company has a security policy specifying that: (i) a piece of information is deemed confidential when its disclosure would harm the interests of the company, and that (ii) confidential information must be protected (and hence cannot be disclosed). Such a policy can be naturally represented by the meta-rule

$$
(\text { Disclose }(x) \rightarrow \text { HarmInterests }) \rightarrow \text { Confidential }(x) .
$$

Now, in this policy, the condition about harming the interests should be represented by an hypothetical expression: an ' $\operatorname{IF}(\ldots)$ Then (...)' rule is the most natural way to represent such a construct. Furthermore, the hypothetical is part of the conditions to define when a piece of information is confidential (actually, 
in this case, is the condition itself). Unfortunately, we cannot use classical material implication $(\supset)$, given the well-known paradoxes of material implication. Consequently, if we model the policy as

$$
(\operatorname{Disclose}(x) \supset \text { HarmInterests }) \rightarrow \text { Confidential }(x),
$$

given the equivalence between Disclose $(x) \supset$ HarmInterests and $\neg$ Disclose $(x) \vee$ HarmInterests, we have the counter-intuitive scenarios where (1) if $x$ is not disclosed then $x$ is confidential (information that is not confidential, no matter if disclosed or not, it does not need to be protected), and (2) if, for any reason, company interests are harmed, then $x$ is confidential, for any piece of information. The policy can neither be defined as

$$
(\operatorname{Disclose}(x) \wedge \text { HarmInterests }) \rightarrow \text { Confidential }(x)
$$

given that a disclosed information (with consequent harm of interest) can no longer be considered confidential. Another situation where meta-rules are useful is when the inclusion of a rule in a set of rules depends upon whether other rules are already in the system. For instance, we can have

$$
r_{1} \rightarrow r_{2},
$$

indicating that the existence of rule $r_{2}$ in the system depends on the existence in the system of rule $r_{1}$. However, typically, such dependencies among rules are stored externally, but if we model them directly into the system using meta-rules, then we can include (or remove) $r_{2}$ automatically, depending on the other rules it depends upon (and thus automating the system's maintenance functionalities). In addition, this feature is beneficial to system integration as it supports context dependant rules. The definition of context dependant policies is valuable in many situations; for instance, the defence forces of a country can have different rules of engagement, depending on the environment in which they are situated. One might think that a simple (and somehow naive) way to achieve this would be to partition the rules into independent rule sets, one for each context, and then to use simpler rules (without nested rules). However, as discussed, there could be dependencies among the rules, and the environments themselves can be defined in terms of rules. Thus, a clear partition of the simple rules might not be feasible.

Meta-rules are useful when a set of rules can provide conditions about other conditions in the same policy. This is the case in legal documents, where often there are provisions conferring power, or delegation, to some agents; in the legal domain, the notion of power is when the legal system allows an agent to create, issue, or revoke, norms affecting other agents. Several works (see $[18,10])$ tried to model such notions using conditional logics since, similarly to hypothetical conditionals, such notions can be faithfully and efficiently represented as rules.

Another area of legal reasoning, where meta-rules proved to be essential to represent the legal processes at hand, is related to the of field of norm change. As we argued, many legislative instruments contain provisions (norms) about who has the power to create, modify, revoke, or abrogate other norms. If norms can be represented as rules [26], and there are norms 'speaking about' other norms, then it would be natural to have rules whose content consists of other rules. 
Different variants of defeasible logic have been proposed $[16,4]$ to incorporate meta-rules in order to describe the logical behaviour of norm changes. An important aspect of norm changes is that some legal systems can specify that specific norms cannot exist (or cannot be in force) in that particular legal system. For example, in the Italian Constitution, Article 27 prescribes that there cannot be norms in the Italian legal system prescribing Capital Punishment. This means that a meta-norm can speak about the positive existence of a rule, as well as preventing a rule to be generated.

To this end, we will distinguish between the content of a rule (which is a binary relationship between a set of premises and a conclusion, both represented as propositions in an underlying, given language), and the name, or identifier, of the rule itself. In this set up, a rule can be understood as a function associating a label to the content of the rule. Similarly, a meta-rule is a function that associates the name, or label, to the content, but in this case the elements of the binary relation corresponding to the content of the meta-rule can contain other rules.

In addition, we will admit negation of rules. If we are able to conclude that a (positive) rule holds, then it means that we can insert the rule (the content of the rule, with a specific name) in the system, and we can use the resulting rule to derive new conclusions. For a negated rule, the meaning is that it is not possible to obtain a rule with that specific content (irrespective of the name).

The paper is structured as follows. In Sect. 2 we introduce a variant of Defeasible Logic able to handle rules and meta-rules and we propose the proof theory of the logic. Then in Sect. 3 we introduce a computationally efficient algorithm to compute the extension of a Defeasible Theory with rules and meta rules and we show that the extension is computable in polynomial time. Finally, we provide some conclusion and a quick discussion on some related work in Sect. 4.

\section{Logic}

Defeasible Logic [1] is a simple and efficient rule-based non-monotonic formalism that proved to be suitable for the logical modelling of different application areas, specifically agents $[19,14,5]$, legal reasoning $[16,4]$, and workflows from a business process compliance perspective $[13,25,24]$. Some of these application fields requires the modelling of contexts and the use of rules in the scope of other rules. Accordingly, extensions of the logic have been developed to capture such features by adopting meta-rules. However, the work on meta-rules in Defeasible Logic focused on defining the extensions of the logic, specifically the proof theoretic features, neglecting to investigate the computational aspects. A major strength of the Defeasible Logic approach, that makes it appealing from the application point of view, is its feasible computational complexity. This paper fills the gap. We start by providing the presentation of the logic from the proof theoretic point of view, and then we will see how to create an efficient algorithm to compute the extension of a defeasible meta-theory.

Let PROP be a set of propositional atoms, and Lab be a set of arbitrary labels (the names of the rules). Accordingly, Lit $=\mathrm{PROP} \cup\{\neg l \mid l \in \mathrm{PROP}\}$ is 
the set of literals. The complement of a literal $l$ is denoted by $\sim l$ : if $l$ is a positive literal $p$ then $\sim l$ is $\neg p$, and if $l$ is a negative literal $\neg p$ then $\sim l$ is $p$. If $\alpha \in$ Lab is a rule label, then $\neg \alpha$ is a rule expression as well, and we use the same convention defined for literals for $\sim \alpha$. We use lower-case Latin letters to denote literals, and lower-case Greek letters to denote rule labels and negated rule labels.

The set of rules is made of two sets: standard rules $R^{S}$, and meta-rules $R^{M}$. A standard rule $\beta \in R^{S}$ is an expression of the type ' $\beta: A(\beta) \hookrightarrow C(\beta)$ ', and consists of: (i) the unique name $\beta \in \mathrm{Lab}$, (ii) the antecedent $A(\beta) \subseteq$ Lit, (iii) an arrow $\hookrightarrow \in\{\rightarrow, \Rightarrow, \sim\}$ denoting, respectively, a strict rule, a defeasible rule and a defeater, (iv) its consequent $C(\beta) \in$ Lit, a single literal. Hence, the statement "All computing scientists are humans" is formulated through a strict rule (as there is no exception to it), whilst "Computing scientists travel to the city of the conference" is instead formalised through a defeasible rule as "During pandemic travels might be prohibited" is a defeater representing an exception to it.

A meta rule is a slightly different concept than a standard rule: (i) standard rules can appear in its antecedent, and (ii) the conclusion itself can be a standard rule. Accordingly, a meta rule $\beta \in R^{M}$ is an expression of the type ' $\beta: A(\beta) \hookrightarrow$ $C(\beta)$ ', and consists of: (i) a unique name $\beta \in \mathrm{Lab}$, (ii) the antecedent $A(\beta)$ is now a finite subset of Lit $\cup R^{S}$, (iii) the arrow $\hookrightarrow$ with the same meaning as for standard rules, and (iv) its consequent $C(\beta) \in$ Lit $\cup R^{S}$, that is either a single literal or a standard rule (meta-rules can be used to derive standard rules).

A defeasible meta-theory (or simply theory) $D$ is a tuple $(\mathrm{F}, R,>)$, where $R=R^{\text {stand }} \cup R^{\text {meta }}$ such that $R^{\text {stand }} \subseteq R^{S}$ and $R^{\text {meta }} \subseteq R^{M}$. F is the set of facts, indisputable statements that are considered to be always true, and which can be seen as the inputs for a case. Rules in $R$ can be of three types: strict rules, defeasible rules, or defeaters. Strict rules are rules in classical sense: every time the premises are the case, so is the conclusion. Defeasible rules represent the non-monotonic part of a defeasible meta-theory as they describe pieces of information that are true under some circumstances, while false or undetermined under others. Accordingly, when the premises of a defeasible rules are the case, so typically is the conclusion but it can be prevented to be the case by contrary evidence. Defeaters are a special type of rules whose only purpose is to defeat contrary statements, but cannot be used to directly draw a certain conclusion. Finally, we have the superiority or preference relation $>$ among rules, which is binary and irreflexive, and is used to solve conflicts. The notation $\beta>\gamma$ means $(\beta, \gamma) \in>$.

Some abbreviations. The set of strict rules in $R$ is $R_{s}$, and the set of strict and defeasible rules is $R_{\mathrm{sd}} . R[X]$ is the rule set with head $X \in\left\{\right.$ Lit $\left.\cup R^{S}\right\}$. A conclusion of $D$ is either a tagged literal or a tagged label (for a standard rule), and can have one of the following forms with the standard meanings in DL:

- $\pm \Delta l$ means that $l \in$ Lit is definitely provable (resp. refuted, or non provable) in $D$, i.e. there is a definite proof for $l$ (resp. a definite proof does not exist);

$- \pm \Delta^{\text {meta }} \alpha, \alpha \in R^{\text {stand }}$, with same meaning as above;

- $\pm \partial l$ means that $l$ is defeasibly provable (resp. refuted) in $D$;

$- \pm \partial^{\text {meta }} \alpha, \alpha \in R^{\text {stand }}$, with the same meaning as above. 
The definition of proof is also the standard in DL. Given a defeasible meta-theory $D$, a proof $P$ of length $n$ in $D$ is a finite sequence $P(1), P(2), \ldots, P(n)$ of tagged formulas of the type $+\Delta X,-\Delta X,+\partial X,-\partial X$, where the proof conditions defined in the rest of this section hold. $P(1 . . n)$ denotes the first $n$ steps of $P$.

Derivations are based on the notions of a rule being applicable or discarded. Briefly, in Standard DL when antecedents are made only by literals, a rule is applicable when every antecedent's literal has been proved at a previous derivation step. Symmetrically, a rule is discarded when one of such literals has been previously refuted. We need to adapt such concepts to deal so that standard rules may appear both in the antecedent, and as conclusions of meta-rules: we thus say that a meta-rule is applicable when each of the standard rules in its antecedent either is in the initial set of standard rules, or has been proved.

Definition 1 (Applicability). Given a defeasible meta-theory $D=(\mathrm{F}, R,>)$, $R=R^{\text {stand }} \cup R^{\text {meta }}$, a rule $\beta \in R$ is \#-applicable, \# $\in\{\Delta, \partial\}$, at $P(n+1)$ iff

1. $\forall l \in \operatorname{Lit} \cap A(\beta) .+\# l \in P(1 . . n)$,

2. $\forall \alpha \in R^{S} \cap A(\beta)$ either (a) $\alpha \in R^{\text {stand }}$, or (b) $+\#^{\text {meta }} \alpha \in P(1 . . n)$.

Notion of discardability is derived by applying the principle of strong negation ${ }^{4}$.

Definition 2 (Discardability). Given a defeasible meta-theory $D=(\mathrm{F}, R,>)$, $R=R^{\text {stand }} \cup R^{\text {meta }}$, a rule $\beta \in R$ is \#-discarded, \# $\in\{\Delta, \partial\}$, at $P(n+1)$ iff

1. $\exists l \in \operatorname{Lit} \cap A(\beta)$. $-\# l \in P(1 . . n)$, or

2. $\exists \alpha \in R^{S} \cap A(\beta)$ such that (a) $\alpha \notin R^{\text {stand }}$ and (b) $-\#^{\text {meta }} \alpha \in P(1 . . n)$

When $\beta$ is a meta-rule and $\alpha$ is not in $R^{\text {stand }}$ (hence $\alpha$ is the conclusion of a metarule), then $\beta$ will stay dormant until a decision on $\alpha$ (of being proved/refuted) is made. The following example is to get acquainted with the concepts introduced.

Example 1. Let $D=(F=\{a, b\}, R, \emptyset)$ be a theory such that

$$
R=\{\alpha: a \Rightarrow \beta ; \quad \beta: b, \beta \Rightarrow \zeta ; \quad \gamma: c \Rightarrow d ; \quad \varphi: \psi \Rightarrow d\} .
$$

Here, both $\alpha$ and $\beta$ are applicable (we will see right below how to prove $+\partial^{\text {meta }} \beta$ ), whilst $\gamma$ and $\varphi$ are discarded as we cannot prove $+\partial c$ nor $\partial^{m e t a} \psi$.

All proof tags for literals are the standard in DL literature [1], and reported here to make the paper self-contained. For this reason, we will omit the negative counterparts as they are straightforwardly obtained from the positive ones by applying the strong negation principle. The definition of $\Delta$ for literals describes forward chaining of strict rules.

$+\Delta l:$ If $P(n+1)=+\Delta l$ then

(1) $l \in \mathrm{F}$, or (2) $\exists \beta \in R_{s}[l]$ s.t. $\beta$ is $\Delta$-applicable.

We now introduce the proof tag for defeasible provability of a literal.

\footnotetext{
4 The strong negation principle applies the function that simplifies a formula by moving all negations to an inner most position in the resulting formula, and replaces the positive tags with the respective negative tags, and the other way around see [15].
} 
$+\partial l:$ If $P(n+1)=+\partial l$ then

(1) $+\Delta l \in P(1 . . n)$, or

(2) (1) $-\Delta \sim l \in P(1 . . n)$, and

(2) $\exists \beta \in R_{\text {sd }}[l]$ s.t. $\beta$ is $\partial$-applicable, and

(3) $\forall \gamma \in R[\sim l]$ then either

(1) $\gamma$ is discarded, or (2) $\exists \varepsilon \in R[l]$ s.t $\varepsilon$ is $\partial$-applicable and $\varepsilon>\gamma$.

We are finally ready to propose the proof tags to prove (standard) rules.

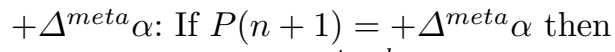

(1) $\alpha \in R^{\text {stand }}$, or (2) $\exists \beta \in R_{s}^{\text {meta }}[\alpha]$ s.t. $\beta$ is $\Delta$-applicable.

A standard rule is strictly proven if either (1) such a rule is in the initial set of standard rules, or (2) there exists an applicale, strict meta-rule for it.

$+\partial^{\text {meta }} \alpha$ : If $P(n+1)=+\partial^{\text {meta }} \alpha$ then

(1) $+\Delta^{\text {meta }} \alpha \in P(1 . . n)$, or

(2) (1) $-\Delta^{\text {meta }} \sim \alpha \in P(1 . . n)$, and

(2) $\exists \beta \in R_{\mathrm{sd}}^{\text {meta }}\left[\left(\alpha: a_{1}, \ldots, a_{n} \hookrightarrow c\right)\right]$ s.t.

(3) $\beta$ is $\partial$-meta-applicable, and

(4) $\forall \gamma \in R^{\text {meta }}\left[\sim\left(\zeta: a_{1}, \ldots, a_{n} \hookrightarrow c\right)\right]$, then either

(1) $\gamma$ is $\partial$-meta-discarded, or

(2) $\exists \varepsilon \in R^{\text {meta }}\left[\left(\chi: a_{1}, \ldots, a_{n} \hookrightarrow c\right)\right]$ s.t.

(1) $\chi \in\{\alpha, \zeta\},(2) \varepsilon$ is $\partial$-meta-applicable, and (3) $\varepsilon>\gamma$.

A standard rule $\alpha$ is defeasibly proven if it has previously strictly proven (1), or (2.1) the opposite is not strictly proven and (2.2-2.3) there exists an applicable (defeasible or strict) meta-rule $\beta$ such that every meta-rule $\gamma$ for $\sim \zeta(A(\alpha)=$ $A(\zeta)$ and $C(\alpha)=C(\zeta)$ ) either (2.4.1) $\gamma$ is discarded, or defeated (2.4.2.3) by (2.4.2.1-2.4.2.2) an applicable meta-rule for the same conclusion $c$. Note that in Condition 2.3 we do not impose that $\alpha \equiv \zeta$, whilst for $\gamma$-rules we do impose that the label of the rule in $C(\gamma)$ is either $\alpha$ or $\zeta$.

$-\partial^{m e t a} \alpha$ : If $P(n+1)=-\partial^{\text {meta }} \alpha$ then

(1) $-\Delta^{\text {meta }} \alpha \in P(1 . . n)$, and either

(2) (1) $+\Delta^{\text {meta }} \sim \alpha \in P(1 . . n)$, or

(2) $\forall \beta \in R_{\mathrm{sd}}^{\text {meta }}\left[\left(\alpha: a_{1}, \ldots, a_{n} \hookrightarrow c\right)\right]$ then

(3) $\beta$ is $\partial$-meta-discarded, or

(4) $\exists \gamma \in R^{\text {meta }}\left[\sim\left(\zeta: a_{1}, \ldots, a_{n} \hookrightarrow c\right)\right]$ s.t.

(1) $\gamma$ is $\partial$-meta-applicable, and

(2) $\forall \varepsilon \in R^{\text {meta }}\left[\left(\chi: a_{1}, \ldots, a_{n} \hookrightarrow c\right)\right]$ then

(1) $\chi \notin\{\alpha, \zeta\}$, or (2) $\varepsilon$ is $\partial$-meta-discarded, or (3) $\varepsilon \ngtr \gamma$.

Given a defeasible meta-theory $D$, we define the set of positive and negative conclusions of $D$ as its meta-extension:

$$
E(D)=\left(+\Delta,-\Delta,+\Delta^{\text {meta }},-\Delta^{\text {meta }},+\partial,-\partial,+\partial^{\text {meta }},-\partial^{\text {meta }}\right),
$$

where $\pm \#=\{l \mid l$ appears in $D$ and $D \vdash \pm \# l\}$ and $\pm \#^{\text {meta }}=\left\{\alpha \in R^{S} \mid \alpha\right.$ appears as consequent of a meta-rule $\beta$ and $\left.D \vdash \pm \#^{m e t a} \alpha\right\}, \# \in\{\Delta, \partial\}$.

Let us propose two theories to explain how the derivation mechanism works. 
Example 2. Let $D=(\mathrm{F}=\{a\}, R,>=\{(\zeta, \chi)\})$ be a theory such that

$$
\begin{aligned}
R^{\text {stand }} & =\{\alpha: a \Rightarrow b, \quad \beta: b \Rightarrow \sim c, \quad \zeta: \sim c \Rightarrow \sim d, \quad \chi: a \Rightarrow d\}, \\
R^{\text {meta }} & =\{\gamma:(\alpha: a \Rightarrow b) \Rightarrow c\} .
\end{aligned}
$$

As $a \in \mathrm{F}$, we prove $D \vdash+\Delta a$, which in cascade give us $D \vdash+\partial a$ ( $\beta$ is hence $\partial$-applicable). Since $\alpha \in R^{\text {stand }}, \alpha$ is $\partial$-applicable and $D \vdash+\partial b$. Moreover, $D \vdash+\Delta^{\text {meta }} \alpha$ and $D \vdash+\partial^{\text {meta }} \alpha$, which makes in turn $\gamma$ being $\partial$-applicable. We conclude with both $D \vdash-\partial c$ and $D \vdash-\partial \sim c$, as the superiority does not solve the conflict between $\beta$ and $\gamma$. The dormant $\chi$ and $\zeta$ can now be considered: $\chi$ is $\partial$-applicable whereas $\zeta$ is $\partial$-discarded. Thus, $D \vdash+\partial d$.

Example 3. Let $D=(\mathrm{F}=\{a, c, d, g\}, R,>=\{(\beta, \gamma)(\zeta, \eta)\})$ be a theory where

$$
\begin{aligned}
R^{\text {stand }} & =\{\alpha: a \Rightarrow b, \quad \zeta: g \Rightarrow \sim b\}, \\
R^{\text {meta }} & =\{\beta: c,(\alpha: a \Rightarrow b) \Rightarrow(\eta: d \Rightarrow b), \quad \gamma: d \Rightarrow \sim(\chi: d \Rightarrow b)\} .
\end{aligned}
$$

As $a, c, d$ and $g$ are facts, we strictly and defeasibly prove all of them. Hence, $\alpha, \zeta, \beta$ and $\gamma$ are all $\partial$-applicable. As before, $\alpha \in R^{\text {stand }}$, thus $D \vdash+\Delta^{\text {meta }} \alpha$ and $D \vdash+\partial c$ make $\beta$ being $\partial$-applicable as well. As $\beta>\gamma$, we conclude that $D \vdash+\partial^{m e t a} \eta$, but we prove also $D \vdash-\partial^{\text {meta }} \chi$ (by Conditions 2.4 and 2.4.1 of $\left.-\partial^{\text {meta }}\right)$. Again, $d$ being a fact makes $\eta$ to be $\partial$-applicable. $\zeta$ has been dormant so far, but it can now be confronted with $\eta$ : since $\eta$ is weaker than $\zeta$, then $D \vdash+\partial \sim b$ (and naturally $D \vdash-\partial b$ ).

The logic presented above is coherent and consistent. This means that given a defeasible meta-theory $D$ : (a) it is not possible to establish that a conclusion is, at the same time, proved and refuted, and (b) if we have positive defeasible proofs for a conclusion and its complement, then the inconsistency depends on the strict (monotonic) part of the theory. This is formally stated in next Proposition 1, which follows from the adoption of the strong principle to formulate the definitions of the proof conditions for positive/negative pairs of proof tags [15].

Proposition 1. Let $D$ be a theory. There is not literal $p$, or label $\alpha$, such that (a) $D \vdash+\# p$ and $D \vdash-\# p$, for $\# \in\left\{\Delta, \Delta^{\text {meta }}, \partial, \partial^{\text {meta }}\right\}$.

(b) If $D \vdash+\partial p$ and $D \vdash+\partial \sim p$, then $D \vdash+\Delta p$ and $D \vdash+\Delta p$; if $D \vdash+\partial^{\text {meta }} \alpha$

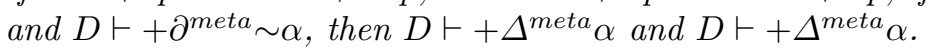

\section{Algorithms}

The algorithms presented in this section compute the meta-extension of a defeasible meta-theory. The main idea being to compute, at each iteration step, a simpler theory than the one at the previous step. By simpler, we mean that, by proving and disproving literals and standard rules, we can progressively simplify the rules of the theory itself. We remind the reader that, roughly said, a rule is applicable when everything in its antecedent has been proved. Note that, trivially, a rule with empty antecedent is always applicable, as there is nothing to prove. Symmetrically, a rule is discarded if (at least) one of the antecedent's 
element has been previously rejected. When a rule is discarded, it can no longer play part in neither supporting its conclusion, nor rejecting the opposite.

Let us consider the theory proposed in Example 2, and let us assume that, at iteration $j$, the algorithm proves $+\partial b$. At the next iteration $j+1, \beta$ will be modified according to what discussed above, and will be $\beta: \emptyset \Rightarrow \sim c(\beta$ is thus applicable). Later on, at iteration $k$, the algorithms prove $-\partial \sim c$, and then proceed in removing $\chi$ from the set of the potentially applicable rules (as $\chi$ is $\partial$-discarded according to Definition 2) and the tuple $(\zeta, \chi)$ from the superiority, as $\chi$ can no longer play any part in supporting $\sim d$.

According to these observations, during the run of the algorithms, every time that a literal or a standard rule is proven, we can remove it from all the antecedents where it appears in. A rule thus becomes applicable when we have removed all the elements from its antecedent. On the contrary, whenever a literal or a standard rule is rejected, we can remove all the rules where such an element appears in the antecedent, as those rules are now discarded. We can also remove all the tuples of the superiority relation containing such discarded rules. The idea of these simplifications is taken from $[12,14]$.

As discussed in Section 2, a meta-rule is applicable when each standard rule in its antecedent is either in the initial set of rules (i.e., in $R^{\text {stand }}$ ), or has been proved later on during the computation and then added to the set of standard rules. This it the reason for the support sets at Lines 1 and 2: $R_{a p p l}$ is the rule set of the initial standard rules, $R^{\alpha C}$ is the set of standard rules which are not in the initial set but are instead conclusions of meta-rules. As rules in $R^{\alpha C}$ are proved/disproved during the algorithms' execution, both these sets are updated.

At Line 3, we populate the Herbrand Base (HB), which consists of all literals that appear in the antecedent, or as a conclusion of a rule. As literals not in the Herbrand base do not have any standard rule supporting them, such literals are already disproved (Line 4). For every literal in HB, we create the support set of the rules supporting that particular conclusion (Line 6), and we initialise the relative set used later on to manage conflicts and team defeater (Line 7).

We need to do the same for those labels for standard rules that are conclusions of a meta-rule. First, if a label for standard rule is neither in the initial set of standard rules, nor a conclusion of a meta-rules, then such a rule is disproved (Line 8). We assume such sets to have empty intersection, as previously motivated. Second, the following loop at Lines 17-20 initialises three support sets: $R[\alpha]$ contains the meta-rules whose conclusion is $\alpha, R[\alpha]_{\text {opp }}$ contains the metarules attacking $\alpha$ ( $\gamma$-like rules in $\left.\pm \partial^{\text {meta }}\right)$, while $R[\alpha]_{\text {supp }}$ contains the meta-rules supporting $\alpha$ ( $\varepsilon$-like rules in $\left.\pm \partial^{m e t a}\right)$.

The following for loop takes care of the factual literals, as they are proved without any further computation. We assume the set of facts to be consistent. Analogously, loop at Lines 17-20 does the same for rules in the initial set of standard rules that may appear in the antecedent of meta-rules.

The algorithm now enters the main cycle (Repeat-Until, Lines 21-40). For every literal $l$ in HB (Lines 23-29), we first verify whether there is a rule supporting it, and, if not, we refute $l$ (Line 24). Otherwise, if there exists an applicable rule $\beta$ supporting it (if at Line 25), we update the set of defeated rules supporting the opposite conclusion $R[\sim l]_{\text {infd }}$ (Line 26). Given that $R[\sim l]$ contains the $\gamma$ rules supporting $\sim l$, and given that we have just verified that $\beta$ for $l$ is 


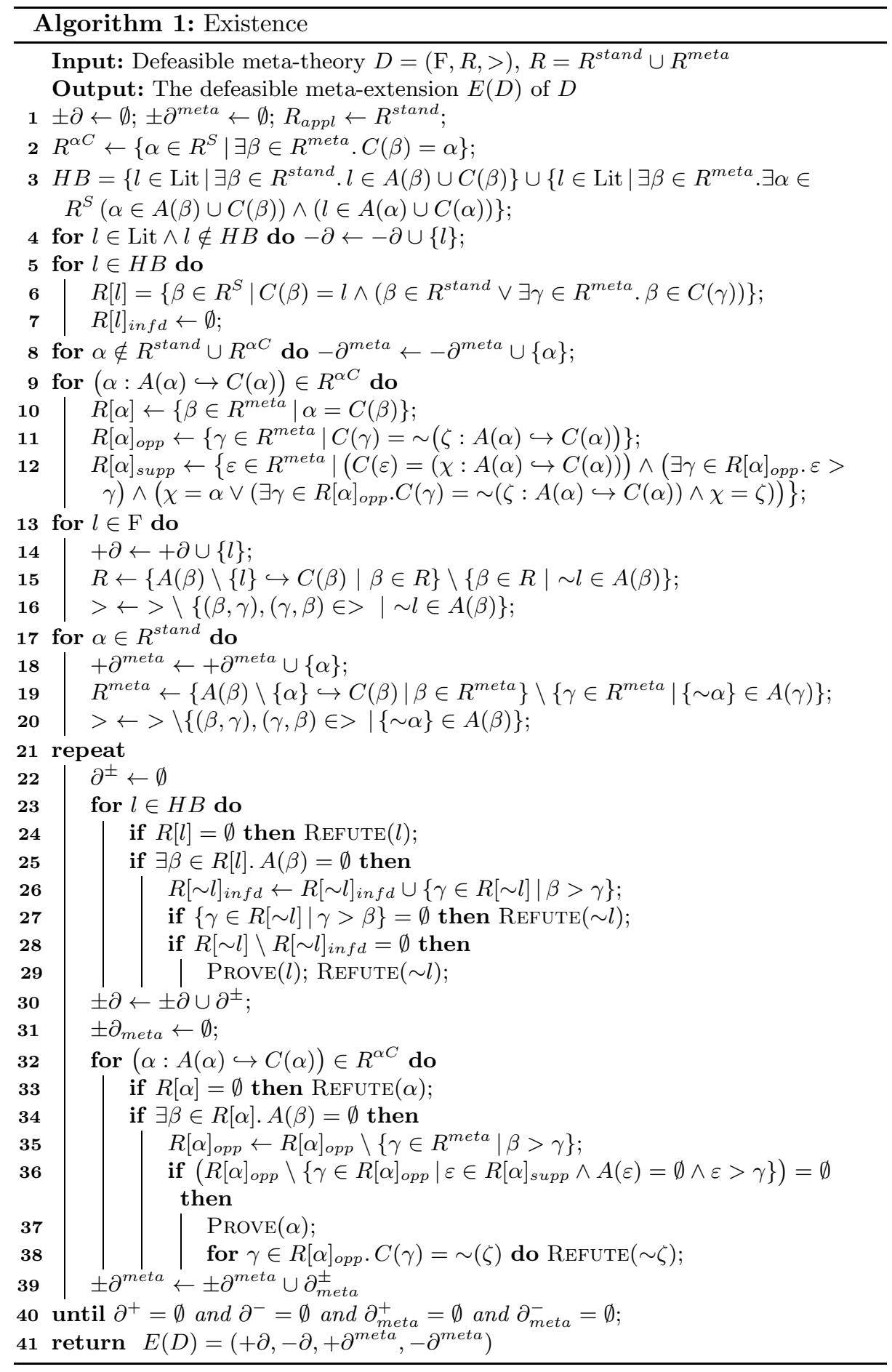


applicable, we store in $R[\sim l]_{\text {infd }}$ all those $\gamma \mathrm{s}$ defeated by $\beta$. The next step is to check whether there actually exists any rule supporting $\sim l$ stronger than $\beta$ : if not, $\sim l$ can be refuted (Line 27 ).

The idea behind the if at Lines 28-29 is the following: if $D \vdash+\partial l$, eventually the repeat-until cycle will have added to $R[\sim l]_{\text {infd }}$ enough rules to defeat all (applicable) supports for $\sim l$. We thus invoke Prove on $l$, and Refute on $\sim l$.

Similarly, when we prove a rule instead of a literal, but we now use $R[\alpha]_{o p p}$ and $R[\alpha]_{\text {supp }}$ in a slightly different way than $R[l]_{\text {inf } d}$, to reflect the differences between $+\partial$ and $+\partial^{\text {meta }}$. Every time, a meta-rule $\beta$ for $\alpha$ is applicable (if at Lines 34-38), we remove from $R[\alpha]_{o p p}$ all the $\gamma \mathrm{s}$ defeated by $\beta$ itself (Line 35). If now there are enough applicable $\varepsilon$ rules supporting $\alpha$ (if check at Line 36), then: (i) we prove $\alpha$, and (ii) we refute all $\zeta$ rules conclusion of $\gamma$ rules in $R[\alpha]_{\text {opp }}$.

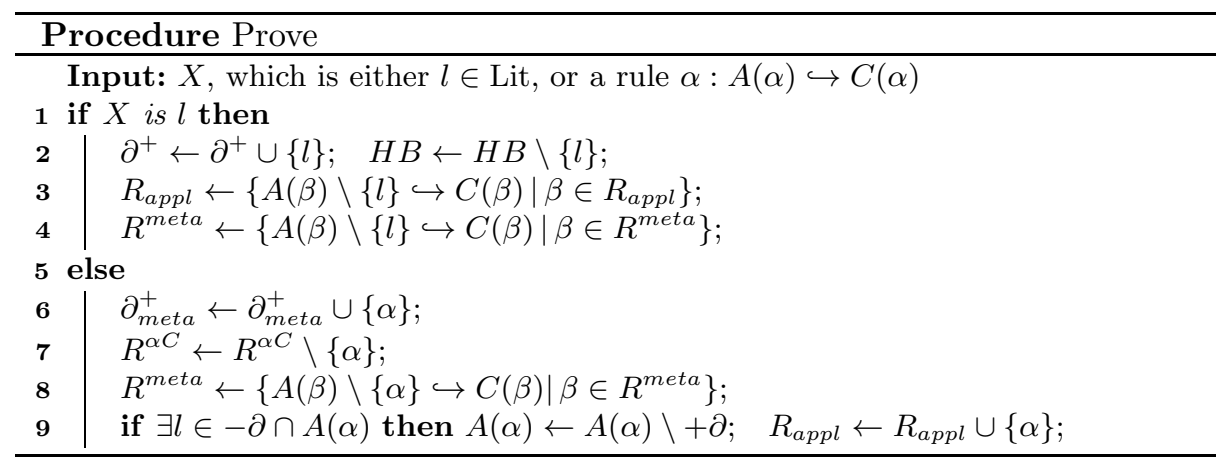

Procedure Prove is invoked when a literal or a standard rule is proved. In case of a literal, we simplify the rules of the theory following what said at the beginning of this section. In case of a rule, we also need to verify whether any of the literal in its antecedent has been already refuted (if check at Line 9). If this is the not case, we can proceed in simplifying $\alpha$ 's antecedent, and then in adding $\alpha$ to the set of standard rules to be evaluated to be applicable.

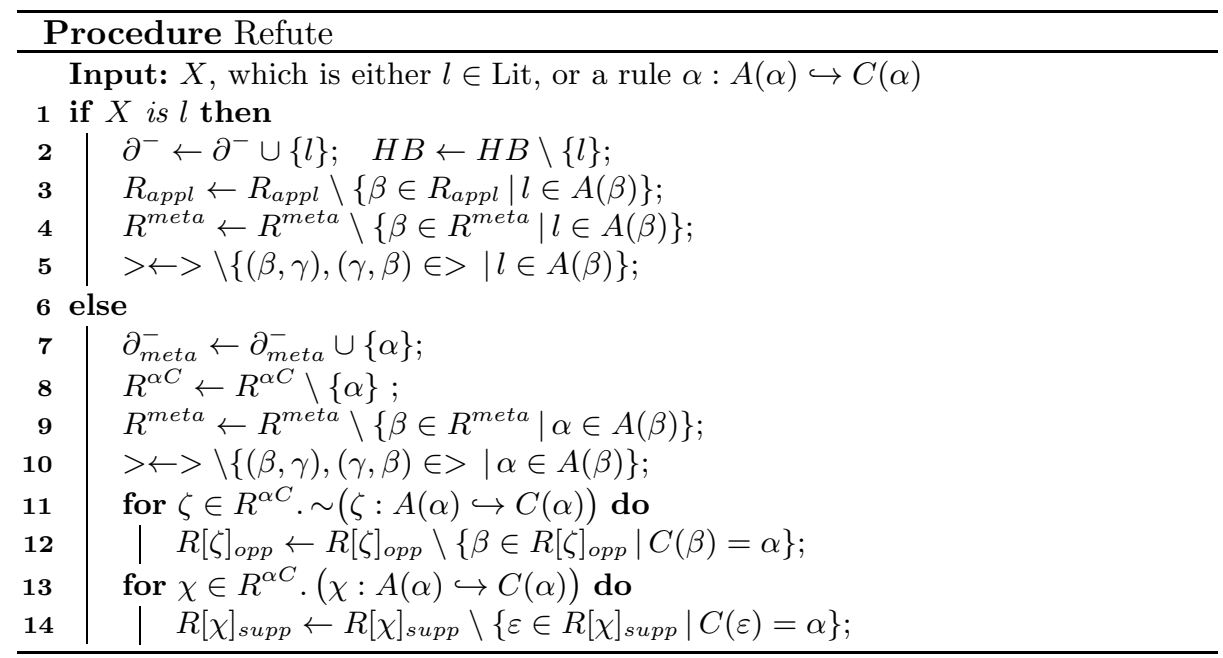


Procedure REFUTE is invoked when a literal or a standard rule is refuted. Again, in case of literals, the simplification operations are the ones detailed in the beginning of this section. In case of rules, the differences are in the two loops at Lines $11-12$ and $13-14$. The former loop updates $R[\zeta]_{o p p}$, as $\beta$ s for $\alpha$ no longer support the counter-argument; symmetrically, the latter loop updates $R[\chi]_{\text {supp }}$.

\subsection{Computational properties}

We discuss the computational properties of Algorithm 1 Existence. Due to space reasons, we only sketch the proofs by providing the motivations of why our algorithms are sound, complete, terminate, and leave out the technical details.

In order to discuss termination and computational complexity, we start by defining the size of a meta-theory $D$ as $\Sigma(D)$ to be the number of the occurrences of literals plus the number of occurrences of rules plus 1 for every tuple in the superiority relation. Thus, the theory $D=(\mathrm{F}, R,>)$ such that $\mathrm{F}=\{a, b, c\}$, $R^{\text {stand }}=\{(\alpha: a \Rightarrow d),(\beta: b \Rightarrow \sim d)\}, R^{\text {meta }}=\{(\gamma: c \Rightarrow(\zeta: a \Rightarrow d))\}$, $>=\{(\zeta, \beta)\}$, has size $3+6+5+1=15$.

Note that, by implementing hash tables with pointers to rules where a given literal occurs, each rule can be accessed in constant time. We also implement hash tables for the tuples of the superiority relation where a given rule appears as either of the two element, and even those can be accessed in constant time.

Theorem 1. Algorithm 1 EXISTENCE terminates and its complexity is $O\left(\Sigma^{2}\right)$.

Proof. Termination of Procedures Prove and Refute is straightforward, as the size of the input theory is finite, and we modify finite sets. The complexity of Prove is $O(\Sigma)$, whereas of Refute is $O\left(\Sigma^{2}\right)$ (two inner for loops of is $O(\Sigma)$ ).

Termination of Algorithm 1 Existence is bound to termination of the repeat-until cycle at Lines $21-40$, as all other cycles loop over finite sets of elements of the order of $O(\Sigma)$. Given that both $H B$ and $R^{\alpha C}$ are finite, and since every time a literal or a rule is proved/refuted, they are removed from the corresponding set, the algorithm eventually empties such sets, and, at the next iteration, no modification to the extension can be made. This proves the termination of Algorithm1 Existence.

Regarding its complexity: (1) all set modifications are in linear time, and (ii) the aforementioned repeat-until cycle is iterated at most $O(\Sigma)$ times, and so are the two for loops at lines $23-29$ and $32-38$. This would suggest that the repeat-until cycle runs in $O\left(\Sigma^{2}\right)$. A more discerning analysis shows that the complexity is actually $O(\Sigma)$ : the complexity of each for cannot be considered separately from the complexity of the external loop (they are strictly dependent). Indeed, the overall number of operations made by the sum of all loop iterations cannot outrun the number of occurrences of the literals or rules $(O(\Sigma)+O(\Sigma))$, because the operations in the inner cycles directly decrease, iteration after iteration, the number of the remaining repetitions of the outmost loop, and the other way around. This sets the complexity of Algorithm1 Existence to $O\left(\Sigma^{2}\right)$.

Theorem 2. Algorithm 1 EXISTENCE is sound and complete, that is 
1. $D \vdash+\partial p$ iff $p \in+\partial p$ of $E(D), p \in$ Lit

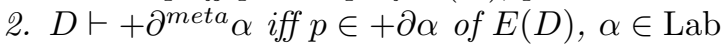

3. $D \vdash-\partial p$ iff $p \in-\partial p$ of $E(D), p \in$ Lit

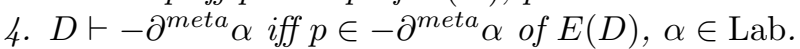

Proof. The aim of Algorithm 1 Existence is to compute a defeasible metaextension of the input theory through successive transformations on the set of facts, rules and the superiority relation. These transformations act in a way to obtain a simpler theory while retaining the same extension. By simpler theory we mean a theory with less symbol in it. Note that if $D \vdash+\partial l$ then $D \vdash-\partial \sim l$, and that if $D \vdash+\partial^{m e t a} \alpha$ then $D \vdash-\partial^{m e t a} \gamma$, with $C(\gamma)=\sim C(\alpha)$. Suppose that the algorithm proves $+\partial l$ or $+\partial \alpha$ (meaning that $l \in+\partial$ or $\alpha \in+\partial^{\text {meta }}$ ). Accordingly, we remove $l$ or $\alpha$ from every antecedent where it appears in, as by Definition 1, the applicability of such rules will not depend any longer on $l$ or $\alpha$, but only on the remaining elements in their antecedents. Moreover, we can eliminate from the set of rules all those rules with $\sim l$ or $\gamma$ in their antecedent (with $C(\gamma)=\sim C(\alpha)$ ), as such rules are discarded by Definition 2 (and adjust the superiority relation accordingly). Finally, when we prove $+\partial \alpha$, then $\alpha$ becomes active in supporting its conclusion and rebutting the opposite.

The proof follows the schemata of the ones in $[12,14]$, and consists in proving that the meta-extension of the original theory $D$ and the meta-extension of the simpler theory $D^{\prime}$ are the same. Formally, suppose that $D \vdash+\partial l$ (symmetrically $D \vdash+\partial^{\text {meta }} \alpha$ ) at $P(n)$. Thus, if $R^{\prime}$ of $D^{\prime}$ is obtained from $R$ of $D$ as follows

$$
R^{\prime}=R_{\text {appl }} \leftarrow\{A(\beta) \backslash\{l / \alpha\} \hookrightarrow C(\beta) \mid \beta \in R\} \backslash\{\beta \in R \mid \sim l / \sim \gamma \in R\},
$$

and if $>^{\prime}$ of $D^{\prime}$ is obtained from $>$ of $D$ as follows

$$
>^{\prime}=>\backslash\{(\beta, \zeta),(\zeta, \beta) \mid \sim l \in A(\zeta) \text { or } \sim \gamma \in A(\zeta)\}
$$

with $A(\gamma)=A(\alpha)$ and $C(\gamma)=C(\alpha)$, then for every $\in$ Lit and every $\chi \in$ Lab

$-D \vdash \pm \partial p$ iff $D^{\prime} \vdash \pm \partial p$, and

$-D \vdash \pm \partial^{m e t a} \chi$ iff $D^{\prime} \vdash \pm \partial^{m e t a} \chi$

The proof that the transformation above produces theories equivalent to the original one is by induction on the length of derivations and contrapositive.

\section{Conclusions and related work}

The topic of this paper is the efficient computation of rules from meta-rules. In general, the topic of how to use (meta-)rules to generate other rules has received little attention. Some exceptions are $[16,4]$ on the use of meta-rules for norm modifications, and [27] which is specifically dedicated to a logic for deriving rules from meta-rules. However, none these works investigate the computationally complexity, nor address the issue of defining algorithms for their logics.

The large majority of the studies that have made use of meta-rules have focused upon the usage of these as a means to determine the scope of rule application, or the result of the application of the rules. In particular, we can identify two research lines: Logic Programming, and Meta-logic. 
Logic programming studies investigated the issue of enhancing the expressivity by allowing nested expressions [21,20]. Nevertheless, these approaches are based on the so called Lloyd-Toper transformation, that transforms nested expressions in (equivalent) logical expressions. Similarly, in [17] disjunctive DATALOG is enriched with nested rules; however, such nested rules, potentially, can be eliminated by using (stratified) negation, but these are kept because they allow for a more natural correspondence with the natural language description of the underlying problem. We have seen in Sect. 1 that this approach suffers from some problems, and it is not appropriate for many uses of meta-rules, in particular when the aim is to represent meta-rules as means to derive rules. Some papers (e.g., [9]) extended logic programming with negation with nested hypothetical implications plus constraints or negation as failure. Specifically, they consider rules with conditional goals in the body, but not implications in the head, and study some goal directed proof procedures.

The notion of meta-rules and close concepts, including meta-logic [3] and meta-reasoning [7], have been employed widely in Logic Programming [2] but also outside it, specifically in context theory [11]. In general, we can look at these studies as methodologically coherent with the notion of hierarchical reasoning, where it is devised a method to choose which reasoning process is more appropriate for the specific scenario in which the process is employed. A specific line of research (strictly connected with the studies in the semantics of Logic Programming) is the Answer Set Programming (ASP) and preferences [8]. Further on, many studies on ASP where meta-rules took place. However, these investigations have not focusing upon nested rules.

A line of work considering the generation of rules from other rules is the work on Input/Output logic (IOL) [22]. The idea of IOL is to define a set of operations on input/output rules (where an input/output rule is a pair $(x, y)$, where $x$ and $y$ are formulas in a logical language) to derive new input/output pairs. Differently to what we do (1): IOL does not consider nested rules, and (2) the derivation mechanism depends on the properties of the operations on which the variant of IOL is defined, and not on the rules on which the logic operates.

A field of investigation that has strongly employed meta-rules, but in a sense that is indeed similar to the one of the theory of contexts, is argumentation. The basic concept derived by the combination of meta-logical structures and argumentation is the metalevel argumentation [23]. Applied metalevel has been investigated in the view of developing a framework where, for instance, admissibility of arguments, and other issues in this field, are dealt with [6].

The problem of nested rules in non-monotonic frameworks from a computational complexity viewpoint deserves a deeper study, and this paper fills this gap. Currently, the focus was on Defeasible Logic without modal operators and temporal expressions (most of the work on meta-rules considers combinations of such features). The basic version of modal and temporal variants of the logic computationally feasible. We plan to extend and combine the algorithm presented in this paper with the algorithms for modal and temporal Defeasible Logic and we expect that the complexity results to carry over to the combination. 


\section{References}

1. Antoniou, G., Billington, D., Governatori, G., Maher, M.J.: Representation results for defeasible logic. ACM Trans. Comput. Log. 2(2), 255-287 (2001). https://doi.org/10.1145/371316.371517

2. Azab, K., Habel, A.: High-level programs and program conditions. LNCS 5214 LNCS, 211-225 (2008). https://doi.org/10.1007/978-3-540-87405-8_15

3. Basin, D., Clavel, M., Meseguer, J.: Reflective metalogical frameworks. ACM Transactions on Computational Logic 5(3), 528-576 (2004). https://doi.org/10.1145/1013560.1013566

4. Cristani, M., Olivieri, F., Rotolo, A.: Changes to temporary norms. In: Keppens, J., Governatori, G. (eds.) ICAIL 2017. pp. 39-48. ACM. https://doi.org/10.1145/3086512.3086517

5. Dastani, M., Governatori, G., Rotolo, A., Song, I., van der Torre, L.: Contextual agent deliberation in defeasible logic. In: PRIMA 2007. LNAI, vol. 5044, pp. 98109. Springer, Heidelberg

6. Dupin De Saint-Cyr, F., Bisquert, P., Cayrol, C., Lagasquie-Schiex, M.C.: Argumentation update in yalla (yet another logic language for argumentation). International Journal of Approximate Reasoning 75, 57-92 (2016). https://doi.org/10.1016/j.ijar.2016.04.003

7. Dyoub, A., Costantini, S., De Gasperis, G.: Answer set programming and agents. Knowledge Engineering Review 33(1) (2018). https://doi.org/10.1017/S0269888918000164

8. Eiter, T., Faber, W., Leone, N., Pfeifer, G.: Computing preferred answer sets by meta-interpretation in answer set programming. Theory and Practice of Logic Programming 3(4-5), 463-498 (2003). https://doi.org/10.1017/S1471068403001753

9. Gabbay, D.M., Giordano, L., Martelli, A., Olivetti, N.: A language for handling hypothetical updates and inconsistency. Log. J. IGPL 4(3), 385-416 (1996). https://doi.org/10.1093/jigpal/4.3.385, https://doi .org/10.1093/jigpal/4.3.3 85

10. Gelati, J., Governatori, G., Rotolo, A., Sartor, G.: Normative autonomy and normative co-ordination: Declarative power, representation, and mandate. Artificial Intelligence and Law 12(1-2), 53-81 (2004)

11. Ghidini, C., Giunchiglia, F.: Local models semantics, or contextual reasoning = locality + compatibility. Artificial Intelligence 127(2), 221-259 (2001). https://doi.org/10.1016/S0004-3702(01)00064-9

12. Governatori, G., Olivieri, F., Rotolo, A., Scannapieco, S.: Computing strong and weak permissions in defeasible logic. J. Philos. Log. 42(6), 799-829 (2013). https://doi.org/10.1007/s10992-013-9295-1

13. Governatori, G., Olivieri, F., Scannapieco, S., Cristani, M.: Designing for compliance: Norms and goals. In: RuleML 2011-America. LNCS, vol. 7018, pp. 282-297. Springer (2011). https://doi.org/10.1007/978-3-642-24908-2_29

14. Governatori, G., Olivieri, F., Scannapieco, S., Rotolo, A., Cristani, M.: The rationale behind the concept of goal. Theory Pract. Log. Program. 16(3), 296-324 (2016). https://doi.org/10.1017/S1471068416000053

15. Governatori, G., Padmanabhan, V., Rotolo, A., Sattar, A.: A defeasible logic for modelling policy-based intentions and motivational attitudes. Log. J. IGPL 17(3), 227-265 (2009). https://doi.org/10.1093/jigpal/jzp006

16. Governatori, G., Rotolo, A.: Changing legal systems: legal abrogations and annulments in defeasible logic. Logic Journal of IGPL 18(1), 157-194 (2010) 
17. Greco, S., Leone, N., Scarcello, F.: DATALOG with nested rules. In: Dix, J., Pereira, L.M., Przymusinski, T.C. (eds.) LPKR Workshop 1997. LNCS, vol. 1471, pp. 52-65. Springer (1997). https://doi.org/10.1007/BFb0054789

18. Jones, A.J.I., Sergot, M.J.: A formal characterisation of institutionalised power. Log. J. IGPL 4(3), 427-443 (1996). https://doi.org/10.1093/jigpal/4.3.427

19. Kravari, K., Bassiliades, N.: A survey of agent platforms. Journal of Artificial Societies and Social Simulation 18(1), 11 (2015). https://doi.org/10.18564/jasss.2661

20. Lifschitz, V., Tang, L.R., Turner, H.: Nested expressions in logic programs. Annals of Mathematics and Artificial Intelligence 25(3), 369-389 (1999). https://doi.org/10.1023/A:1018978005636

21. Lloyd, J.W., Topor, R.W.: Making prolog more expressive. Journal of Logic Programming 1(3), 225-240 (1984). https://doi.org/10.1016/0743-1066(84)90011-6

22. Makinson, D., Van Der Torre, L.: Input/output logics. Journal of philosophical logic 29(4), 383-408 (2000)

23. Modgil, S., Bench-Capon, T.: Metalevel argumentation. Journal of Logic and Computation 21(6), 959-1003 (2011). https://doi.org/10.1093/logcom/exq054

24. Olivieri, F., Cristani, M., Governatori, G.: Compliant business processes with exclusive choices from agent specification. In: PRIMA 2015. LNCS, vol. 9387, pp. 603-612. Springer. https://doi.org/10.1007/978-3-319-25524-8_43

25. Olivieri, F., Governatori, G., Scannapieco, S., Cristani, M.: Compliant business process design by declarative specifications. In: PRIMA 2013. LNCS, vol. 8291, pp. 213-228. Springer. https://doi.org/10.1007/978-3-642-44927-7_15

26. Sartor, G.: Legal Reasoning: A Cognitive Approach to the Law. Springer (2005)

27. Song, I., Governatori, G.: Nested rules in defeasible logic. In: RuleML 2005 Conference, Galway, Ireland. LNCS, vol. 3791, pp. 204-208. Springer 